\title{
NUEVA INSCRIPCIÓN LUSITANA PROCEDENTE DE PORTALEGRE
}

\author{
Blanca M. Prósper y Francisco Villar \\ Universidad de Salamanca \\ indoling@usal.es y fvillar@usal.es
}

\section{NEW LUSITANIAN INSCRIPTION FROM PORTALEGRE}

\begin{abstract}
La aparición de una nueva inscripción lusitana indígena, la más meridional de las conocidas hasta ahora, constituye una novedad de primer orden para el estudio de las lenguas prerromanas de la Península Ibérica. Este artículo aborda el análisis de la inscripción desde el punto de vista sintáctico y etimológico y concluye que su contenido aporta nuevos datos sobre la clasificación del lusitano como dialecto cercano a las lenguas itálicas y el latín.
\end{abstract}

Palabras-clave: Indoeuropeo; epigrafía; lusitano; lenguas itálicas; lenguas celtas.
The recent discovery of the up to now Southernmost indigenous Lusitanian inscription is good news for the study of the Lusitanian language and, more generally, for the knowledge of the IndoEuropean languages of the Iberian Peninsula. This paper aims to offer a whole analysis of the text from the syntactic as well as the morphological and lexical points of view, and adds new evidence in support of the idea that Lusitanian is closely related to Latin and the rest of the italic languages.

Keywords: Indo-European; epigraphy; Lusitanian; Italic languages; Celtic languages.

\section{INTRODUCCIÓN}

El epígrafe que nos ocupa en este trabajo ha aparecido en el valle de la Ribeira da Venda, al norte de Arronches, en el distrito de Portalegre y acaba de ser editado por A. Carneiro et alii $2008^{1}$. Se trata de la más meridional

1 Este artículo se entregó a fines de 2008 (proyecto DGICYT Hum2005-01340). La asistencia al X Coloquio de lenguas y culturas prerromanas (Lisboa, 26-28/2/2009) exigió refinar algunas lecturas. Así se indica cuando no coinciden con la edición y además se deben al consenso de los asistentes, entre los que destacamos al editor y anfitrión, J. d'Encarnação, así como a F. Beltrán, J. Cardim Ribeiro, J. L. García Alonso, J. A. Correa, M. P. GarcíaBellido, M. C. González Rodríguez, J. Gorrochategui, A. Guerra, J. de Hoz, M. P. de Hoz, E. Luján, M. Mayer, J. J. Moralejo, J. L. Ramírez Sádaba, A. Redentor, J. M. Vallejo y J. Velaza. 
de todas las inscripciones indígenas del occidente de la Península Ibérica y de la única aparecida hasta ahora en la frontera misma de la Lusitania Pacensis. Adicionalmente, contiene las menciones más meridionales que se conocen de las divinidades BANDI, REVE (cuyo culto documentado hasta ahora no sobrepasaba el Tajo excepto por algunos ejemplos de Cáceres) y posiblemente de MVNIDI. Por lo demás, hay motivos para situar el derivado toponímico CANTIBIDONE, en la 1.5 del epígrafe, algo al norte del emplazamiento de esta inscripción. Todo apunta a una posible dependencia parcial de este epígrafe de realidades lingüísticas algo más septentrionales de su zona de hallazgo, sobre todo si se admite que la forma CANTI en la 1.9 sea una abreviatura de CANTIBIDONIENSIS. Por lo demás, es imprescindible tener en cuenta la constatación de los editores, que, basándose en criterios paleográficos, datan esta inscripción a comienzos de la ocupación romana de Lusitania. En efecto, la aparición de una «P abierta» o «P silana» apunta a que se puede datar el monumento a mediados o finales del siglo I a. C. Obsérvese que dicha variedad de la P aparece también en Arroyo de la Luz III (cf. Villar y Pedrero 2001).

Se trata de una laja de piedra arenisca tosca y deteriorada por los lados, cuyas medidas son $88 \mathrm{~cm}$ de altura $\times 75 \mathrm{~cm}$ de anchura $\times 3,5 \mathrm{~cm}$ de profundidad. Con seguridad, como sucede con las inscrs. indígenas conocidas (excepto quizá Arroyo de la Luz III), está destinada a conmemorar un sacrificio múltiple. Si en ocasiones anteriores se sacrificaban varias víctimas en honor de las divinidades protectoras de la comunidad, en ésta no caben grandes dudas sobre el carácter institucional o colectivo del evento, dado que la magnificencia de lo ofrendado lo acerca a una hecatombe. A juzgar por el aspecto de la parte superior de la piedra puede haberse perdido algo del texto, aunque es improbable que falte una línea entera. A menos que haya que leer PORCO(S) en la parte desgastada que hay al inicio de la primera línea, esto rompe con la estructura triádica de Cabeço das Fráguas, que describe el sacrificio de un cerdo, una oveja, una oveja añoja y un toro. En este sentido, el contenido es algo más parecido al de otras inscrs. indígenas.

A todos ellos está dedicado este trabajo. Abreviaturas: $\mathrm{NP}=$ antropónimo, $\mathrm{NL}=$ topónimo, $\mathrm{ND}=$ teónimo, inscr. $=$ inscripción. 
MAPA 1.-LAS PRINCIPALES INSCRIPCIONES LUSITANAS

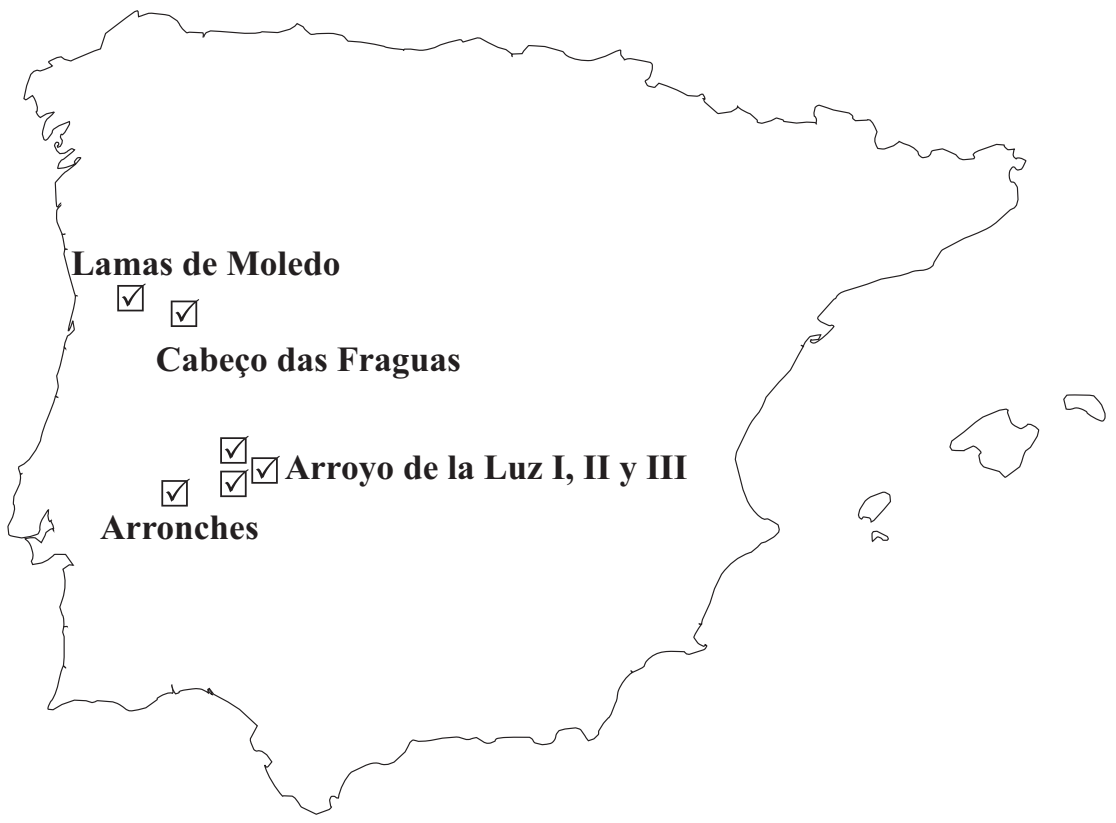

I. El teXto DE LA INSCRIPCIÓN DE ARRONCHES

$$
\begin{aligned}
& \text { [--------]AM }{ }^{2} \cdot \text { OILAM • ERBAM [---] } \\
& \mathrm{HARASE} \bullet \mathrm{OILA} \cdot \mathrm{X} \cdot \mathrm{BROENEIAE} \cdot \mathrm{H}[------] \\
& {[\ldots . .] \text { OILA } \bullet X \cdot \operatorname{REVE~AHARACVI~} \bullet \text { TAV }[---]^{3}} \\
& \text { IFATE }^{4} \cdot \mathrm{X} \cdot \text { BANDI HARACVI AV[---] } \\
& \text { MVNITIE CARIA }{ }^{5} \text { CANTIBIDONE } \bullet\left[-{ }^{6}\right.
\end{aligned}
$$

${ }^{2}$ Eds. [-]...XX. La terminación de acusativo $-<\mathrm{AM}>$ es la apreciada por los asistentes al coloquio.

${ }^{3}$ Eds. T $~ A V$.

${ }^{4}$ Eds. IEATE.

${ }^{5}$ CARIA según la edición. Los asistentes al coloquio han visto CARLA o CAREA, pero la decisión es difícil porque el interior del ángulo que supuestamente formarían los trazos aparece algo erosionado.

${ }^{6}$ En la autopsia se ven restos al final de lo que podría ser un grafo $<\mathrm{A}>$. 


\author{
APINVS • VENDICVS • ERIACAINV[S] \\ OVGVI[-] ANI $^{7}$ \\ $\mathrm{ICCINVI} \bullet \mathrm{PANDITI} \bullet \mathrm{ATTEDIA} \cdot \mathrm{M} \bullet \mathrm{TR}$ \\ PVMPI $・$ CANTI $・$ AILATIO
}

El aspecto de la piedra sugiere que fue preparada para recibir la inscr. y alisada al efecto en sus dos terceras partes superiores, mientras que el tercio inferior fue dejado sin desbastar quizá con el propósito de que fuera ésta la parte que sería introducida en la tierra, de manera que la piedra quedara en pie como una estela. Nuestra propuesta es que la inscr. inicialmente planificada contenía el texto que va desde el inicio hasta OVGVI[-]ANI inclusive. Teniendo en cuenta sólo esas líneas, la estructura de la inscr. resulta comprensible: un primer bloque de cinco líneas que contiene la enumeración de víctimas y divinidades destinatarias. Esta primera parte está redactada en lusitano, aunque no sería descabellado pensar que contuviera ya algunas influencias latinas. A continuación una línea en blanco separa el contenido de la ofrenda de la firma del (de los) ordenante(s) del sacrificio. Las dos líneas siguientes consisten en una fórmula onomástica, la del (de los) oferente(s): «Apinio Vendico Eriaceno», que parece estar redactada en latín. Y ahí concluía la inscr. inicialmente proyectada. La piedra debió de ser clavada en el suelo donde permanecería así algún tiempo. Cabe preguntarse si la extraña forma OVGVI[-]ANI no encubre un etnónimo o localicio, con sufijo -anus o -tanus, en nom. pl., que abarca a varios firmantes.

En un momento posterior, aprovechando el espacio en blanco que había quedado en la piedra entre la última línea inscrita (OVGVI[-] $\underline{\mathrm{ANI}}$ ) y el ras del suelo, se inscribió una segunda dedicatoria, similar a muchas otras de la epigrafía lusitana en que el ND (ICCINVI PANDITI) conserva los rasgos morfológicos de la lengua lusitana, mientras que la formula onomástica del dedicante (en esta ocasión una mujer) tiene morfología latina: «Attedia M. TR. hija (?) de Pumpio Cantio (o cantibidonense)». A continuación hay una forma AILATIO que recuerda lógicamente al NP Aelatius.

Un aspecto de la teonimia de este epígrafe, que parece avalar la conclusión de que contiene dos textos diferentes redactados en dos momen-

7 Eds. OVOVIANI. El grafo $<\mathrm{G}>$ ha sido el apreciado por la mayoría de los asistentes, aunque en efecto es un trazo vertical que se extiende más de lo esperable. 
tos sucesivos, es que, mientras en la primera parte se vincula el epíteto HARACVI/AHARACVI a una serie de divinidades de alcance regional muy amplio, como son BANDI, REVE y MVNIDI/MVNITIE (con la excepción de BROENEIAE), las divinidades de la segunda parte, ICCINVI y PANDITI, son desconocidas hasta el momento, lo que apunta a su carácter local; no se conmemora expresamente que se les hayan realizado ofrendas animales de ninguna clase y carecen de vinculación toponímica explícita. Entre ambas dedicatorias se dan diferencias epigráficas, referentes a la forma de algunas letras y posiblemente también a la profundidad de las incisiones, que podrían denotar una segunda mano, o un segundo momento de empleo de la misma mano.

\section{ANÁLISIS E INTERPRETACIÓN DEL TEXTO}

Como en el caso de las demás inscrs. indígenas de Lusitania, se trata de un sacrificio animal, ofrecido a una serie de divinidades. Y, como sucede en concreto con la inscr. rupestre del Cabeço das Fráguas (Sabugal, Guarda), su parte central se compone de secuencias de acusativo (objeto directo) seguido de dativo (objeto indirecto), que vienen a expresar sencillamente los animales sacrificados seguidos de las divinidades a las que se sacrifican. Como en el caso mencionado, no se emplea un verbo en forma personal, sino que se supone implícitamente ${ }^{8}$.

\section{[--------] AM OILAM ERBAM [---] / HARASE}

Después de la parte perdida, que seguramente es un ac. sg. fem., del que apenas nada puede decirse, el primer animal que aparece como ofrenda en la parte conservada del texto es OILAM ERBAM, que parece una secuencia de sustantivo + adjetivo concertando en ac. sg. fem., con el orden de palabras esperable en una lengua SVO, el mismo que se documenta en las otras inscrs.

8 Durante el Coloquio, J. Cardim Ribeiro se expresa a favor de pensar que las secuencias son de dativo + acusativo, lo que es contrario a la praxis de otros sacrificios de la zona (excepto quizás el de Freixo de Numão) y exige entender que en la primera parte de la inscripción hubo un dativo teonímico. Pero notemos que no puede descartarse que haya en realidad un verbo inicial con sujeto implícito. 
lusitanas. Hasta ahora, la palabra OILAM, que fue interpretada convincentemente por A. Tovar 1967 como IE *owi-lā, diminutivo de *owi- 'oveja', se documentaba solamente en Cabeço das Fráguas, donde aparecen los sintagmas OILAM y OILAM VSSEAM. Tovar interpretaba VSSEAM como un adjetivo *uts-yo- 'añojo', procedente de *wetos 'año'.

En nuestro caso, ERBAM se deja interpretar con facilidad como procedente de IE * $H_{1} e r-b(h)_{o-}$ 'pardo' (cf. IEW, p. 334). Aunque probablemente estamos ante un derivado antiguo en -bho- (a su vez producto de la lexicalización y extensión a partir de un núcleo de antiguos compuestos cuyo segundo elemento es ${ }^{*}-b_{h} H_{2}-o$ - 'brillar'), algunas lenguas, quizá por motivos expresivos, muestran restos inequívocos de /b/ primitiva. Es el caso de germánico *erp- en aisl. iarpr 'marrón', aaa. erpf 'parduzco', etc. (en cualquier caso,

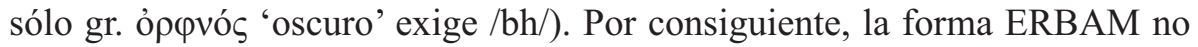
puede considerarse diagnóstica de la representación de aspiradas en lusitano. Notemos que en celta se conserva una forma parecida en airl. erb 'vaca', que $I E W$, p. 326, hace proceder de un étimo *er(i)- 'Bock, Schaf, Kuh', y establece una distinción artificial con el étimo anterior, teniendo en cuenta que no es nada raro que, por un fenómeno trivial de antonomasia, los animales reciban su denominación por su color. No parece seguro que aquí estemos ante una enumeración de sustantivos referidos a una serie de animales diferentes, y parece probable que se esté haciendo una distinción entre razas o variedades ovinas que existe hasta hoy'.

Con esto se aclara algo el significado de ERBA de Arroyo de la Luz I, en la secuencia SECIAS ERBA MVITIEAS ARIMO PRAESONDO SINGEIETO. Parece claro que ERBA se refiere al sacrificio de un animal identificado por su color, en este caso posiblemente con omisión del sustantivo correspondiente. Teniendo en cuenta las etimologías probables de SECIAS (*sek-yo'parte cortada') y MVITIEAS (*moi-ty-ayo- o *moi-ty-o- 'mutuo, donado, intercambiado') cabría especular con la posibilidad de que se haya omitido el grafo $-<\mathrm{S}>$ de genitivo (o se haya perdido fonéticamente /s/) ante consonante (los casos de $-<\mathrm{S}>$ en Arroyo de la Luz son antevocálicos) y entonces se puede traducir el objeto directo de esta oración como 'los cortes (la carne cortada) de la (oveja) parda ofrecida en sacrificio', como sus sinónimas itálicas, umbro pruseçias y latín prōsiciae.

\footnotetext{
9 Nótese a este respecto el posible paralelo de $C I L \mathrm{II}^{2}$, 14, 292 (Sagunto, Valencia): DIANAE MAXIMAE / VACCAM OVEM ALBAM PORCAM...
} 
HARASE es, según todas las apariencias, la divinidad a la que se hace la ofrenda de la oveja parda. Como vamos a ver, parece que ese nombre está relacionado con AHARACVI y con HARACVI. Pero, no obstante la identidad de su radical, existen diferencias en la sufijación. También es notable la circunstancia de que AHARACVI / HARACVI sea un epíteto de las divinidades conocidas REVE y BANDI, mientras que HARASE aparece sin ND. Sólo hay dos vías de explicación de este fenómeno: bien es un epíteto sin ND, bien existe un ND entre OILAM ERBAM y HARASE. En el primer caso la dificultad es patente; la omisión del ND se da cuando el contexto cultural permite al lector suplir su ausencia porque se trata de una divinidad conocida, protectora del poblado o de un grupo humano. Pero en nuestro caso no parece probable que haya una divinidad por excelencia de la zona, seguida de otras dos que tienen idéntico ámbito de influencia pero que son mencionadas por su nombre. Por consiguiente, parece más probable que a la forma HARASE le preceda el ND propiamente dicho, perdido con el enorme desgaste de la parte superior derecha de la piedra. Este desgaste está bien probado por el hecho de que en las 11. 3 y 4 las palabras TAV[-] y AV[-] han perdido varias de sus letras, pero también porque en la 1.2 tenemos una secuencia más de ND más epíteto, BROENEIAE $\mathrm{H}$, que, si no se admite que se ha perdido la parte derecha de la línea, contiene la única abreviatura de este epíteto de base har- que aparece a lo largo de la inscr. Desde el momento en que en la foto se constata que el desgaste de la piedra va en disminución a partir de la 1.1 , se deduce que en las 11.1 y 2 había originalmente sitio para varias letras. Resumiendo, HARASE, H[---], HARACVI y AHARACVI cumplen la misma función en el texto, que es acompañar a otros tantos ND.

Finalmente, la vacilación inicial entre HARACVI y AHARACVI sugiere que la aspiración se había perdido fonéticamente tras la adopción del alfabeto y su escritura correcta era mera cuestión de conocimiento del uso gráfico. Esto es compatible tanto con una etimología con velar aspirada /gh/ como con /s/ aspirada (cf. infra), que permitiría aventurar una etimología *sorā de IE *ser- 'fluir' 10 .

\footnotetext{
${ }^{10}$ Alternativamente habría que pensar que se trata de ND «asociados» mediante la adición del sufijo velar a una localidad *Haro-, $-\bar{a}$, excepto en el caso de REVE, dios-río, que es mencionado habitualmente por su propio nombre con sufijo velar endocéntrico. En este caso, sería un compuesto hipostático de ad- y el NL *Harā, es decir, '(río) que pasa por *Harā', lo cual es tipológicamente más raro.
} 
OILA

A continuación, y en dos ocasiones consecutivas, se ofrece OILA X. Teniendo en cuenta la reiteración de la numeración romana en esta inscr., es obvio que aquí se ofrecen igualmente ovejas, y en concreto dos veces, a dos divinidades distintas, en número de diez. Todo lo cual puede querer decir una cosa: que OILA es un ac. pl. *owilāns, que ha dado lusitano *oilās, y que el dialecto de esta zona pierde la silbante en posición final absoluta probablemente a través de un estadio intermedio de aspiración, como sucede por ejemplo en el dialecto español de Andalucía y Extremadura, y como sucedió en su momento en el área oriental del dominio del latín. Acaso el genitivo ERBA de Arroyo de la Luz I sea otra manifestación del mismo fenómeno, limitado allí a la posición anteconsonántica (siendo esa misma posición la única que nos es dado constatar en Arronches) ${ }^{11}$. Esto significa, adicionalmente, que estamos ante un sacrificio de considerable envergadura, donde se ofrecen las víctimas por decenas.

\section{BROENEIAE}

Hace unos años, en su edición de Arroyo de la Luz III, F. Villar y R. Pedrero sugirieron interpretar las formas en $-<\mathrm{AE}>\mathrm{y}-<\mathrm{A}>$ de Arroyo de la Luz I no como grafías alternativas para el dat. fem., sino respectivamente como un locativo y un dativo. Esto es plausible en la medida en que la aparición de dats. en $-<\mathrm{A}>$ puede explicarse como fenómeno analógico del correspondiente masculino y no como producto de una evolución fonética, solución que sería igualmente aplicable a los dats. en $-<\mathrm{A}>$ del latín rústico (cf. Villar 1986). Si fuera así, podríamos explicar las formas HARASE y BROENEIAE como locativos. Aunque esto solucionaría diversos problemas (sobre HARASE, cf. infra), lo cierto es que con ello se pierde coherencia y uniformidad textual, en la medida en que se hablaría por una parte de sacrificios localizados en diversos lugares y por otra de sacrificios ofrecidos a divi-

\footnotetext{
${ }^{11}$ Ambos ejemplos de OILA, así como IFATE, sintácticamente acs. pl., van seguidos del numeral latino X, que a su vez va seguido de BRONEIAE, REVE y BANDI. Tanto si se toma en cuenta el numeral decem como las divinidades que siguen al numeral, la posición de la -s era anteconsonántica. J. Cardim Ribeiro adelanta la ingeniosa hipótesis de que se trate de interpunciones en aspa, que genera sus propios problemas.
} 
nidades diversas, sin relación entre una cosa y la otra. Por lo tanto, parece a priori más aconsejable prescindir de esta interpretación morfológica y asumir que, en este sistema al menos, existe cierta vacilación en la representación de los dats. fem., quizá porque, siendo una inscr. particularmente primitiva, el cambio estuviera in fieri. No obstante, se constata que la grafía generalizada del presente documento para el segundo elemento de un diptongo es sistemáticamente $-<\mathrm{I}>$. Tenemos por lo tanto OILAM, ERIACAINVS, AILATIO. Esto hace sospechoso el segmento final de BROENEIAE, aunque la presencia de $-y$ - y su aparición en sílaba final son condicionantes adicionales que deben ser tenidos en cuenta. Más abajo exploraremos otra posible adscripción de la terminación de esta forma a propósito de HARASE y de MVNITIE.

BROENEIAE es difícil de interpretar etimológicamente. Quizá la vinculación más atractiva sea con la forma alargada $* b h r(e) H_{l}-u$-, con metátesis *bhr(e)uH $H_{1^{-}}$'hervir, borbotar', etc. (véase Schrijver 1991, pp. 253-254), de donde *bhre/ouH $H_{1}$-én-yo- > *browén-yo- > *broén-yo-. Cf. ai. bhurváni- 'agitado, salvaje', de *bhrH $H_{l} u$-én-i-, y, tal vez, la diosa latina Fur(r)ina de las aguas subterráneas. El resultado de la labial puede estar condicionado al contexto o bien proceder de un dialecto, celta o no, con el resultado /bh/ > /b/. El conjunto BROENEIAE H[---] se entendería como una típica fórmula bimembre lusitana de derivado teonímico en -yo-, aquí 'divinidad de la fuente', uel sim., seguido del epíteto que contiene su nombre o expresa su relación con una localidad.

Hay otro aspecto de esta inscr. que invita a la reflexión sobre el problema de los dats. fem., y es la aparición de un dat. CARIA/CAREA. Hasta ahora, la documentación de esta terminación parecía distribucionalmente incluida en la de los dativos en $-<\mathrm{O}>\mathrm{y}-<\mathrm{V}>$. Así, la norma gráfica de Lamas de Moledo tenía $-<\mathrm{AI}>/-<\mathrm{OI}>$, y en cambio las de Cabeço das Fráguas y Arroyo de la Luz tenían $-<\mathrm{A}>/-<\mathrm{O}>$. A la luz de la hipótesis del carácter «imitativo» de los dats. fem. en $-<\mathrm{A}>$, obtenía coherencia el dato que arrojaba un estudio geográfico de la variación de las dos desinencias. En efecto, en $L R P$ (p. 392) se trataba de mostrar que en la zona galaica esta imposición (de una forma masculina única $-<\mathrm{O}>$ ) no habría ido seguida por la de un dat. fem. en $-<\mathrm{A}>$, a diferencia de lo ocurrido en el área meridional. La distribución de $-<\mathrm{A}>$ coincide grosso modo con la de los dats. temáticos en $-<\mathrm{V}>$. En cambio $-<\mathrm{OE}>,-<\mathrm{OI}>$ se acerca a la de $-<\mathrm{AI}>, \mathrm{y}-<\mathrm{VI}>$ ocupa una posición intermedia. Por tanto, habría que ver la zona meridional como innovadora en el dat. fem., generalizando siempre las formas $\sin -i$, es decir $-<\mathrm{A}>\mathrm{y}-<\mathrm{O}>\mid-<\mathrm{V}>$; la zona central como 
conservadora con $-<\mathrm{AE}>/-<\mathrm{AI}>$, por una parte, $\mathrm{y}-<\mathrm{O}>/-<\mathrm{OI}>(-<\mathrm{V}>,-<\mathrm{VI}>)$, por otra, y la zona del norte como conservadora en el femenino $-<\mathrm{AI}>$ e innovadora en el masculino $-<\mathrm{O}>12$.

Como se adelantaba en $L R P$, cuando hablamos de innovación en la generalización de $-<\mathrm{O}>/-<\mathrm{V}>$, esto vale tanto si partimos de una pérdida fonética de $-i$ en una única desinencia primitiva *-ōi, como si aceptamos una situación más compleja, la defendida por Villar y Pedrero 2001, en el sentido de que el lusitano heredó de una fase anterior ambos dativos, de los que el más antiguo desde una perspectiva IE sería - $\bar{o}$ (como puede aceptarse sin problemas si tenemos en cuenta que en osco y umbro tenemos respectivamente los dats. -úi /-e, y en latín arcaico epigráfico DVENOI) y que luego, ya en Hispania, diversos dialectos que no estamos en condiciones de delimitar con precisión generalizaron resultados alternativos o mantuvieron ambos ${ }^{13}$. Pero ahora ya conocemos una inscr. donde aparece un dat. fem. en $-<\mathrm{A}>$, probablemente otro en $-<\mathrm{AE}>$ (aunque veremos que existen explicaciones alternativas) $\mathrm{y}, \mathrm{sin}$ lugar a dudas, no menos de tres masculinos en $-<\mathrm{VI}>$. En realidad, éstos son fáciles de integrar en la visión que se daba en $L R P$, basada en buena medida en la dependencia que muestran los sistemas notacionales de las inscrs. individuales de las fechas de la progresiva implantación del alfabeto latino, que hipotéticamente habría avanzado de sur a norte. Se decía allí que los dats. en $-<\mathrm{V}>$, que no faltan en la zona central, también mostraban una distribución muy meridional, junto con $-<\mathrm{O}>$, mientras que los dats. en $-<\mathrm{VI}>$ ocupaban más bien una zona intermedia, donde alternan con las soluciones $-<\mathrm{V}\rangle,-<\mathrm{O}>$ $\mathrm{y}-<\mathrm{OI}>/-<\mathrm{OE}>$.

En aquel momento se entendía por meridional el escaso material que en efecto venía de allí, y que se limitaba casi en exclusiva al área central de la Lusitania Emeritensis, y en concreto a Cáceres. Para ser ésta una zona amplia y precozmente romanizada, era evidente que la carencia de $-i$ era primitiva

${ }^{12}$ F. Villar 1981, pp. 164-168; 1985, pp. 44-46, interpreta -ō en el dativo temático IE como un antiguo directivo de origen adverbial que aparece en el directivo hetita $-a$, en el adverbio latino quo y en los griegos óv $\omega$, кó $\tau \omega$. La presencia de una desinencia $-i$ en todos los demás dativos y sobre todo en los femeninos (-āi) hizo que la analogía llevara en numerosas lenguas a una situación más regular, bien creando la desinencia masc. *-ōi tradicionalmente reconstruida, bien generando la variedad de los dats. fem. en $-\bar{a}$.

${ }^{13}$ Nieto 1993 defendía la tesis de que estamos ante una mera vacilación gráfica, producto de la detención en un estadio relativamente temprano del proceso de monoptongación y cierre vocálico del diptongo -āi en zonas no urbanas. 
en los dos dativos. Sin embargo, estamos ahora ante la inscr. indígena más meridional conocida, y más occidental que las de Cáceres. Por tanto hay que reformular las relaciones anteriores, en el sentido de que $-<\mathrm{VI}>$ ocupa no sólo la zona intermedia, sino la más meridional, y por tanto traza un vector antes inexistente que une esta zona con la Lusitania central, desplazando el sistema de Arroyo de la Luz con sus particularidades al extremo más oriental de Lusitania.

Además este epígrafe pone en cuestión la relación aceptada entre los dativos de los dos géneros. Aquí, la existencia de un dat. en $-<\mathrm{A}>$ como CARIA/ CAREA no parece depender de la de $-\langle\mathrm{O}\rangle /-\langle\mathrm{V}\rangle$, sino que se combina en un mismo texto con tres casos de $-<\mathrm{VI}>$. Esto indica que, si originalmente alternaban ambos dats. masc., la desaparición de $-i$ puede haber sido más precoz en el femenino y no al contrario. $\mathrm{O}$, aplicando la propuesta reconstructiva de Villar, resultaría que un dat. masc. primitivo en $-\bar{o}$ habría condicionado la aparición del dat. en - $\bar{a}$, que se habría impuesto a $-\bar{a} i$, que habría quedado limitada a la función de locativo (en la que el masculino tenía de suyo -oi), sin que dejaran de alternar para el masculino las soluciones $-<\mathrm{O}\rangle,-<\mathrm{V}\rangle,-<\mathrm{OI}\rangle$, $-<\mathrm{VI}>$ como pasa en el centro de Portugal. La distribución de $-<\mathrm{A}>$ abarca, por consiguiente, en todo caso el centro y sur de Portugal y el suroeste de España y es compatible en general tanto con $-<\mathrm{O}>\mathrm{y}-<\mathrm{V}>$, como ya sabíamos por las grandes inscrs. indígenas y los teónimos aislados, como con $-<\mathrm{VI}>$, cosa que por vez primera descubrimos en el ejemplo de CARIA/CAREA, si es que efectivamente se trata de un dat. en $-\bar{a}$. No olvidemos el caueat impuesto por nuestra ignorancia: si CARIA/CAREA fuera una abreviatura de CARIA(CAE), la forma BROENEIAE se convertiría en firme candidata a entenderse como dat. fem. en $-<\mathrm{AE}>$. Entonces el sistema gráfico de esta inscr. tendría sólo dats. con $-i$ final para los tres géneros. Sobre todo ello volveremos más abajo.

\section{REVE AHARACVI}

La divinidad masculina REVE, identificada por Villar 1996 como referida a un curso de agua, es una formación atemática procedente probablemente de ${ }^{*} H_{I} r e u-$. Sus apariciones se extienden por toda la fachada occidental, aunque éste es el caso más meridional conocido. Como en todos los casos documentados hasta ahora, la desinencia de dat. masc. IE *-ei aparece grafiada como 
$-<$ E $>$. Esto, que en sí mismo parece irrelevante, contrasta vivamente con el hecho de que otros dats. atemáticos del mismo origen muestren vacilaciones diversas, como sucede con la divinidad BANDI, que, sobre todo en el área lusitana central y meridional, aparece con terminación en $-<\mathrm{E}>,-<\mathrm{EI}>\mathrm{e}-<\mathrm{I}>$. La razón podría relacionarse con los diferentes orígenes y direcciones de expansión del culto a una y otra divinidad. Pero en cualquier caso se esperaría una representación más uniforme de la misma desinencia para la misma zona y para palabras diversas. La solución puede estar en otro sitio: en la existencia de dos resultados condicionados de *-ei en final de palabra: [r:] en todos los casos excepto tras $-w$-, en que la pronunciación es [e:]. Si el primero era variante sincrónica de /i:/, la pertenencia a fonemas diferentes explicaría la diferente elección gráfica a pesar de la presión morfológica, como en esta misma inscr. si se acepta que $-<\mathrm{E}>\mathrm{y}-<\mathrm{VI}>$ (en HARASE y HARACVI respectivamente, cf. infra) son variantes del morfema original $-\bar{o} i$.

MAPA 2.-Distribución y VARIANTES DE REVE

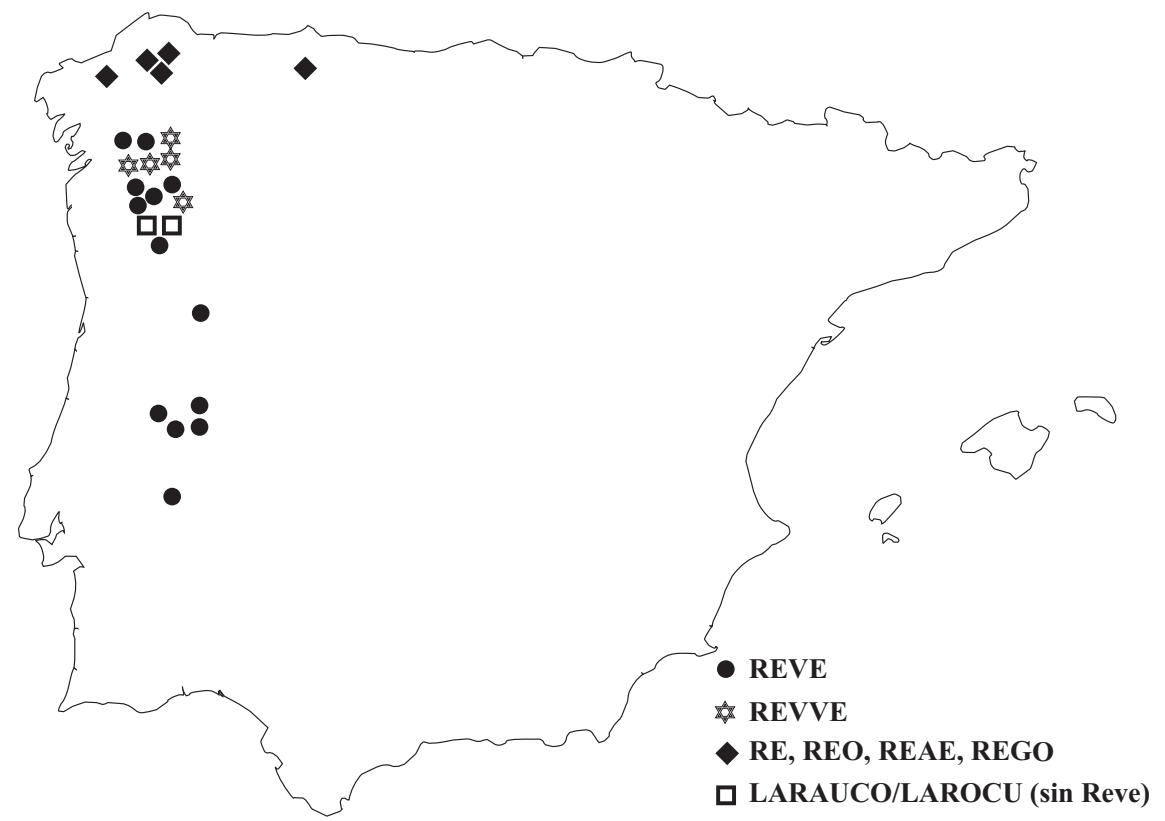


Mapa 3.-Los distintos epítetos de REVE

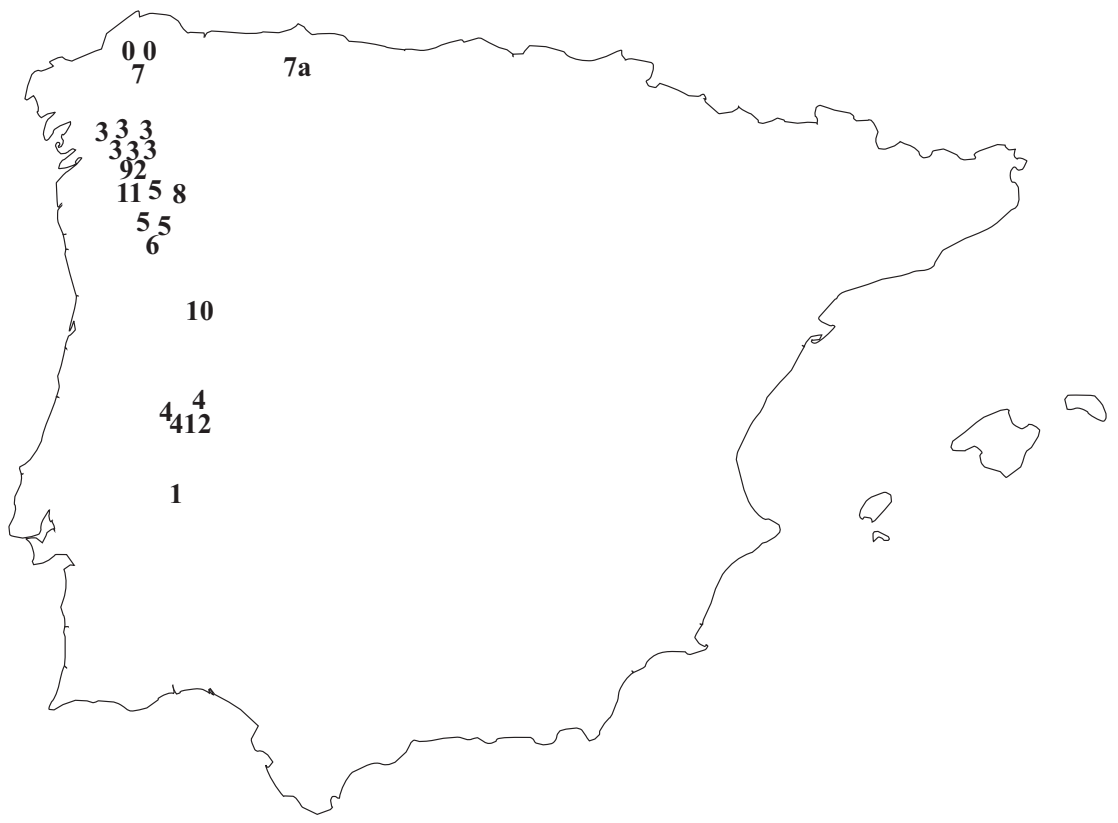

0) Sin epíteto; 1) Aharacui; 2) Amoaego; 3) Ana Baraego, Anabaraego, Anabar, Anabar, Anabaraeco, Anabaraego; 4) Langanid[aeco], Langanidaeigui, Langanitaeco; 5) Larauco, Larauco, Larocu; 6) Marandigui; 7) Paramaeco; 7a) Pece Parameco; 8) Reumiraego; 9) Siboico; 10) Trasanciace; 11) Tre[; 12) Veif; 13) Veisuto.

\section{TAV(---) IFATE}

Una rápida comparación con Cabeço das Fráguas permite constatar la identidad formular entre TAV(---) IFATE y TAVROM IFADEM. Con esto se saldaría la necesidad de leer una interpunción, y por consiguiente una abreviatura, en la secuencia T. AV[---]. Lo que hay entre los caracteres $\langle$ T $>$ $\mathrm{y}<\mathrm{A}>$ se sitúa en un punto algo más elevado que la interpunción que separa AHARACVI de TAV[---], y difícilmente puede ser otra cosa que una mella en la piedra. El sintagma TAV(---) IFATE va seguido del número romano $\mathrm{X}$, lo que indica que es un acusativo de plural. Notemos que es la segunda 
vez que aparece en una inscr. indígena el apelativo *tauro-, con una fonética conservadora y por lo tanto incompatible con la que muestran todas las lenguas celtas, en las que esta forma ha sufrido metátesis, dando *tarwo- y finalmente *tarßo-. Esto incluye celtib. Taruotureska en la tésera de Osma y TARVODVRESCA en otra tésera (cf. Jordán 2005; Villar y Untermann 1999).

B. Prósper 2004 ha propuesto interpretar IFADEM como un nombre de agente compuesto *en(i)-bhat-yo- 'que embiste' (cf. el NP gálata Kov $\beta \alpha \tau \iota \alpha \kappa o \varsigma)$. IFADEM habría sufrido por tanto sonorización de una dental sorda intervocálica - $t$-, fenómeno típico de los dialectos no-celtas del occidente peninsular. La aparición de IFATE confirma este punto, y evidencia que esta inscr. es lo bastante arcaica como para no reflejar la sonorización de las oclusivas sordas. Además, invalida la sospecha de que IFADEM es una forma incompleta (cf. $M L H$ IV, p. 730).

El grafo inicial $<\mathrm{I}>-$ representa el resultado de la preposición IE $* H_{1}$ en-. En la fecha de documentación de nuestros textos más antiguos el lusitano ha experimentado ya la pérdida de nasal ante fricativa sorda, como en la divinidad astur COSSVE, procedente de *kom- $d h H_{1}-t u$ - (LRP, pp. 239-240). El cambio se produjo probablemente con anterioridad a la adopción del alfabeto latino en todas partes. Este fenómeno empieza a revelarse en los errores de las inscrs. latinas arcaicas, que a veces optan por la realidad fónica contra la norma (cf. IFEROS, COSOL). El cierre de [e:] en esa posición está indirectamente avalado por el cierre de [o:] en algunas variantes del ND COSSVE, como CVSVE, indicando que existía una tendencia al cierre de las vocales largas de origen tanto primario como secundario. Esto se fundamenta mejor si se acepta una solución similar para los casos del sufijo -êt- reconstruido para PANDITI y MVNIDI/MVNITIE (cf. infra).

IFATE aparece en lugar del ac. pl. **IFATES. Desde el punto de vista defendido para IFADEM, esto querría decir que la sucesión de cambios fonéticos sería la siguiente: $-y o n s>-i n s>-e n s>-e s /-\bar{e} s>-e h /-\bar{e} h>-e /-\bar{e}$. Por lo demás, la parte perdida por el desgaste de la derecha del texto sugiere que después de TAV[--] no habría más de dos caracteres, lo que implica que la palabra puede completarse como TAV[RO], o TAV[RV], con la desinencia de ac. pl. masc. *-ons. 


\section{BANDI HARACVI}

La divinidad masculina BANDI se documenta bajo diversas formas, de las cuales la más arcaica es, sin duda, BANDVE, documentada fundamentalmente en Galicia. Éste es, como hemos visto con el ejemplo de REVE, su testimonio más meridional. En la Lusitania central y meridional aparece bajo las formas BANDI, BANDEI (Covilhã, Castelo Branco, RAP, núm. 21), BANDE (Castelo de Vila da Feira, Aveiro, $R A P$, núm. 19) y BANNEI (Belver, Portalegre, RAP, núm. 35).

\section{AV[--- MVNITIE CARIA CANTIBIDONE}

La división en segmentos significativos, que permite aislar los sintagmas en dativo que contienen nombres y epítetos divinos, se complica considerablemente en nuestro caso. Pero desde un punto de vista léxico, todas las formas cuentan con correspondencias plausibles.

MVNITIE parece un dat. fem. atemático idéntico al dat. MVNIDI en MVNIDI EBEROBRIGAE TOVDOPALANDAIGAE (Garrovillas, Cáceres), MVN[I]DI. IGAED(ITANAE) (Castelo Branco, RAP, núm. 169), MVNIDI FIDVENEARVM (Paços de Ferreira, Oporto, CIL II, 5607; RAP, núm. 568) y [M]VNIDI (Celorico da Beira, Guarda, RAP, núm. 169a).

En $L R P$, cap. VI.VIII se enumeraban las correspondencias de esta forma, si procede de la raíz *men- 'elevarse' (cf. IEW, p. 726), con alargamiento dental en lat. mons (< *monti-), ai. mati- < *mnti- 'monte', gr. $\mu$ oṽ $\sigma \alpha<$

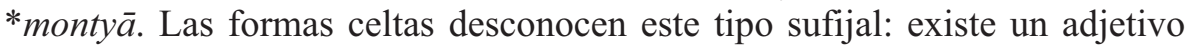
*mon-iyo- en gal. mynydd, córn. meneth, bret. menez 'monte'. En aisl. mønir tenemos la variante con grado largo *mōn-iyo-. Otros paralelos próximos son onomásticos, como en el caso del NL ilirio Movítıov, que Mayer 1957-1959 hacía proceder de *Mōn-et-yo-. Finalmente puede establecerse una relación con Iüno Monèta. Si atendemos a los dos últimos, se diría que tenemos aquí un nombre de agente en -et- con el significado de 'pro-minente, que se eleva o destaca' (cf. Irslinger 2002).

Como es bien sabido, el nominativo de este tipo se caracterizaba por su vocalismo largo. El tipo formacional reseñado se hace proceder de un paradigma alternante con un nom. sg. -et-, ac. sg. -et-, gen., dat., etc., -t-, al que se suele atribuir valor agentivo, y que tal vez se limitaba, en origen, a formas compuestas. No obstante, es relativamente frecuente que los casos disponi- 


\section{Mapa 4.-Testimonios y VARIANTES DE MVNIDI}

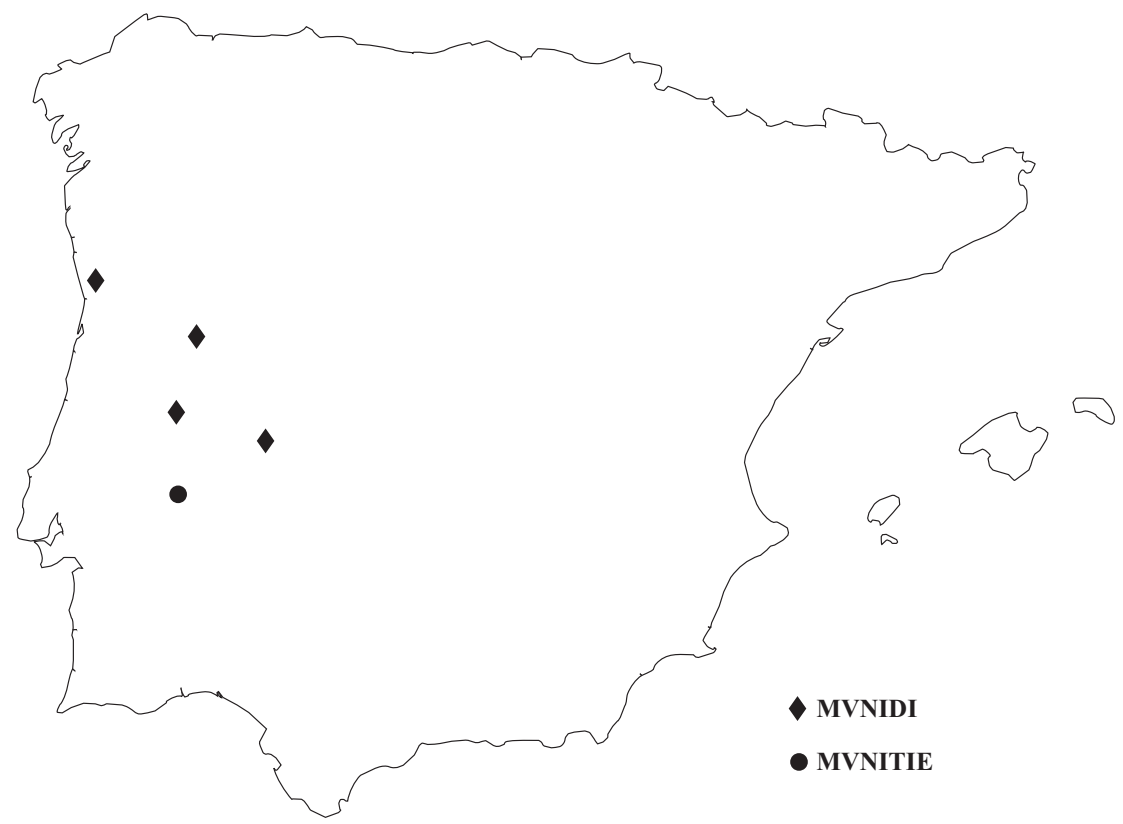

bles de las lenguas hayan generalizado el grado largo del nominativo a todo el paradigma. En efecto, así sucede en algunas formas celtas, como airl. fili, gen. filed 'que ve', en ogámico gen. sg. VELITAS (correspondencia directa del NP de la poetisa germana Veleda), en los dos casos documentados en avéstico, rauuas-carāt- 'que circula libremente' y fra-carāt- 'que se mueve

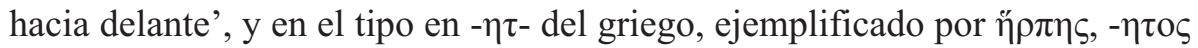
'que repta" ${ }^{14}$. En latín se consideran formas primarias y por tanto antiguas los nombres de objeto stīpes, tudes y el adjetivo teres 'redondo', que muestran grado pleno sufijal generalizado en toda la flexión.

Esto explicaría la secuencia - $<\mathrm{ID}>-,-<\mathrm{IT}>-$, siempre que aceptemos una evolución lusitana /e:/ > /i:/ semejante a la de celta y osco-umbro, y permi-

${ }^{14}$ Cf. Rieken 1999, pp. 86-90. En latín hay formas atribuibles a este tipo flexivo que por su valor pasivo han recibido explicación como nombres de objeto, primitivamente adjetivos: seges, -ětis 'siembra', cf. a.gal. segeticion 'proles', o teges, -etis 'cubierta', cf el NP galo Ciuo-tegetis (CIL XII, 41). 
tiría establecer una conexión muy cercana con la forma latina Monèta. No podemos descartar la posibilidad, por lo demás, dada la escasez de nuestros datos, de que se trate de una evolución condicionada del vocalismo breve -et-, que habría sufrido una tendencia al cierre en sílaba átona. En nuestro caso es muy probable que haya que contar también con vocalismo largo radical /o:/, que ha dado regularmente /u:/ en contacto con nasal labial, como sucede en Arroyo de la Luz con las formas PVPPID, ENVPETANIM.

El sufijo -et- es típico, sobre todo en latín e itálico, de ND, como sucede con galo Mars Loucetius, Leucetius frente a Iuppiter Lücetius (en zona osca), o latín dī indigites (de un sg. *endo-ag-et-s) y, probablemente, salvando las dudas que ofrecen sus respectivas etimologías, la divinidad osca Anagtiai (dat. sg.), o la marsa Angitia, o las divinidades umbras llamadas Açetus (dat. pl. $)^{15}$. Podemos añadir como ejemplo itálico el caso de véneto veskes, dat. sg. vesketei 'alumno', literalmente 'que se alimenta'.

Una última posibilidad, que resultaría admisible tal vez para PANDITI pero es poco convincente para el caso de MVNITIE/MVNIDI, consistiría en reconstruir un compuesto cuyo primer elemento es un adjetivo (véase lo dicho más abajo a propósito de PANDITI) y donde el segundo elemento es, a su vez, un derivado con sufijo dental $*_{-} H_{l} i-t$ - de la raíz $*_{H_{l}} e i$ - 'ir'. Éste es el caso probablemente en lat. comes, -itis y limes, -itis 'que va en diagonal' (limo-) > 'frontera', ai. arthét- 'presuroso', y probablemente no es otra cosa que un subtipo secundario de los temas en -et- originado en una escisión paradigmática muy antigua, en virtud de la cual en algunas formas se generalizó el grado cero de los casos oblicuos, subtipo que forma compuestos masculinos de valor agentivo, como el nutrido grupo de formas del antiguo indio que podemos ejemplificar con la forma deva-stu-t- 'que alaba a los dioses',

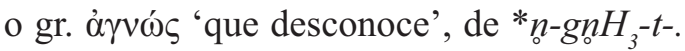

En cualquier caso, se esperaría encontrar algún elemento que sirva para vincular de forma independiente a la divinidad MVNITIE con las elevaciones orográficas. Parece que recibe la ofrenda AV[--- y que a continuación porta dos epítetos, CARIA y CANTIBIDONE, pero la estructura resultante MVNITIE CARIA es comparativamente muy infrecuente. Si se trata de una fórmula trimembre y referida a una única divinidad MVNITIE CARIA CANTIBIDONE, es de suponer que la fórmula estuviera abreviada, y habría que leer algo así como MVNITIE CARIA(CAE?) CANTIBIDONE(--?),

\footnotetext{
${ }^{15}$ Cf. Untermann 2000, pp. 76, 97.
} 
comparable a las dedicaciones de Idanha (Castelo Branco) a ERBINE. IAIDI(?). CANTIBIDONE(NSI?), y que a su vez enlazan con la MVN[I] DI. IGAED(ITANAE) de Idanha. No obstante, el paralelismo dista de ser completo, puesto que ERBINE está documentada como forma teonímica independiente, mientras que MVNITIE muestra una sospechosa diferencia con MVNIDI en su terminación. Volveremos sobre esto más abajo, a propósito de la morfología de MVNITIE. Podría también pensarse que AV[---] es el animal o grupo de animales ofrecido al menos a dos divinidades: una podría ser MVNITIE sin epíteto y la otra CARIA CANTIBIDONE.

Pero un nuevo argumento se opone a las dos ideas anteriores: parece razonable pensar que un sacrificio de diez toros ocuparía el último lugar en el relato de la sucesión de ofrendas, que está, con toda probabilidad, en relación icónica con la sucesión temporal de los hechos que se conmemoran. A esta luz sería poco verosímil que a continuación apareciera una hipotética ofrenda designada por AV[---] al final de la línea, lugar en que, a juzgar por el aspecto que ofrece la parte superior derecha de la piedra, no quedaba sitio para muchos caracteres, lo que parece excluir la presencia de un numeral y contrasta con el sacrificio de diez toros que le precede. El problema de la relevancia y el de la ausencia de interpunciones a partir de la mención de BANDI se salvan aceptando que las divinidades son dos, insertas en sendas fórmulas trimembres, de las que la primera sería BANDI HARACVI AV[---] y la segunda MVNITIE CARIA CANTIBIDONE. Persiste un último obstáculo relacionado con $\mathrm{AV}[---]$, que por su escasa extensión difícilmente puede ser la parte restante de un epíteto divino y que, además, sería el único caso en la inscr. en que a un ND se le añade un segundo epíteto después de HARACVI. Finalmente, MVNITIE podría no ser una divinidad, sino simplemente un adjetivo con el valor de 'de montaña' que modificaría a la ofrenda AV[---], como sucede con TAV[---] IFATE. Esto presenta considerables dificultades morfológicas y obliga a aceptar que está en ac. pl., pero sin numeral añadido. Ninguna de estas soluciones resulta muy satisfactoria.

Alternativamente, podemos pensar que una ofrenda muy considerable, consistente en diez toros, se hizo a un grupo de varias divinidades. Como se ha dicho, entre BANDI HARACVI y el resto de las formas no existe interpunción, lo que podría sugerir que estamos ante tres ND con sus tres epítetos correspondientes: BANDI HARACVI, AV[---] MVNITIE y CARI-A CANTIBIDONE. Desde este punto de vista se podría establecer un curioso paralelo entre CARIA CANTIBIDONE y la dedicación a la divinidad BOREA 
CANTIBEDONIESI que hace un individuo, que por su procedencia étnica es un limicus, en un ara hallada en Moguer (Huelva, CIL II, 4963, a. 27). En $L R P$, p. 173, se entendía la base subyacente al adjetivo *bor-(i)yo-como * $g^{w}$ oro-, $-\bar{a}$ 'montaña', forma que se continúa más o menos directamente en

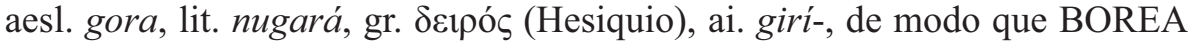
CANTIBEDONIESI sería 'a la (divinidad) del monte Cantibedonio'. CARIA o CAREA CANTIBIDONE sería, estrictamente, la 'divinidad de la roca Cantibedonia', y no resulta impensable que el referente externo sea el mismo para ambos casos. El origen de CARIA/CAREA es evidente: procede de * $k r$ - 'cortar', que da numerosas formaciones con el valor genérico de 'roca, piedra'; cf. el epíteto CAIRIOCIEGO (Tuy, Pontevedra) o el NL CARIENSI (Zamora).

La variación $<\mathrm{E}>$, $<\mathrm{I}>$ en el interior de CANTIBIDONE y la aparente abreviación de este localicio se documentan también en las dos inscrs. de Idanha (Castelo Branco) ya mencionadas más arriba, que están dedicadas a una ERBINE. IAEDI./IAIDI(TANAE?). CANTIBIDONE(NSI?). Como se puso de manifiesto en $L R P$, pp. 215-219, la forma que subyace a un localicio Cantibidonensis es *kanti-bhedho-, forma con etimología y tratamiento fonético celta de las oclusivas sonoras aspiradas, convertidas en sonoras, que se debe interpretar como uso toponímico del apelativo compuesto que designa una cantera, y tiene un parentesco cercano con celtib. arkatobezom 'mina de plata' (K.0.7).

De esta forma, CARIA/CAREA CANTIBIDONE sería una de las conocidas fórmulas bimembres cuyo primer elemento, habitualmente considerado como el ND, es un apelativo alargado por el sufijo relacional básico -yo-, y CANTIBIDONE la forma, completa o abreviada, que adopta el nombre de la localidad a la que se adscribe, es decir, el epíteto. Directamente comparables serían CARNEO CALANTICENSI (Evora) o VORDIO TALACONIO (Guarda). En realidad, como se expone en $L R P$, son muy pocos los epítetos divinos que no van dotados de un sufijo velar o de equivalentes como -ensis, -itanus, y cuando esto sucede suele tratarse de formas polisilábicas o que emplean sufijos complejos preferidos en otras zonas, como VORDIO TALACONIO o COSO CALAEVNIO.

Naturalmente, se puede prever la objeción por parte de quienes niegan la existencia de dats. fem. lusitanos en $-<\mathrm{A}>\mathrm{y}$, consiguientemente, consideran probado que las formas terminadas en $-<\mathrm{A}>$ de las inscrs. indígenas son necesariamente nominativos femeninos (cf. $M L H$ IV). Aunque la disputa 
se salda con diversos argumentos, en el caso que nos ocupa puede añadirse cuando menos otro más: un NP CARIA no se documenta en ninguna parte de la fachada occidental, a diferencia de lo que sucede con las formas de ambos géneros CARVS (aunque su genitivo de singular resulta lógicamente ambiguo) y CARA, que puede ser de raigambre tanto indígena como latina. La única posible excepción es el ciudadano Lucius Aemilius Cario de Cáparra (Cáceres). Se localizan formas del derivado Caricus en el área celtibérica y sus alrededores (véanse los detalles de la distribución geográfica en Vallejo 2005, pp. 260-261). La forma CARLA sólo se conoce como NL en Arroyo de la Luz.

En otro orden de cosas, la distribución escogida para el texto no favorece esta idea: si una individua llamada Caria/Carla Cantibidone(nsis) fuera la responsable de un sacrificio de envergadura semejante, su posición en la inscr. se encontraría fuertemente destacada, lo que dista mucho de poder explicar por qué a continuación aparecen uno o varios individuos masculinos ocupando una línea completa bien separada de lo anterior. En otras palabras, si tanto APINVS VENDICVS ERIACAINVS como ATTEDIA son NP, habría que figurarse que enmarcan una segunda dedicación a ICCINVI PANDITI, produciendo una estructura incoherente.

Por otra parte, en MVNITIE, la extraña grafía $-<\mathrm{IE}>$, sin paralelos exactos conocidos, puede deberse a una vacilación en la representación del resultado de IE *-ei, que en el área occidental aparece notado como $-<\mathrm{I}>,-<\mathrm{EI}>\mathrm{y}$ $-<\mathrm{E}>$. Hay un único caso de representación anómala, $-<\mathrm{IAE}>$ del ND BANDI, que se documenta en un epígrafe de Brozas (Cáceres) dedicado a BANDIAE APOLOSEGO (Salas et al. 1989). Para Pedrero 1999, p. 541, se trata de un error por BANDI, lo que no descartamos a la vista de la proliferación meridional de formas corruptas. No olvidemos que la inscr. que tenemos entre manos en este trabajo es antigua y en más de un aspecto arcaizante, y que su representación de BANDI es la esperable en esta zona, de modo que toda equiparación entre BANDIAE y MVNITIE corre el riesgo de ser ficticia. En segundo lugar, puede que se trate de un fenómeno esporádico de hipercaracterización. En tal circunstancia se trataría de un tema en -i-, con generalización del grado cero sufijal a todo el paradigma y nueva adición de la desinencia $-e i$. Esto requiere que tanto MVNITIE como MVNIDI sean temas en $-i$-. Su etimología probable sería entonces *moni-ti-, nombre de acción en -ti- cuya base no es la raíz, sino un tema verbal de presente causativo *mon-eyo-, que ha tendido a sustituirla incluso en formas heredadas. Esto encontraría un 
paralelo en formas celtibéricas que se atribuyen a *men- 'pensar', como el NP monituukoos de la tésera de Sasamón (Burgos, K.14.1), el ara dedicada a las MATRIBVS MONTVCINIS (Salas de los Infantes, Burgos) o el nombre de acción monimam de Tiermes (Soria, K.11.1-2) y Julióbriga (Cantabria, K.26.1 $)^{16}$.

Podemos plantear, sin embargo, una hipótesis alternativa que abre interesantes perspectivas a la interpretación de la secuencia AV[--- MVNITIE como ND + epíteto derivado, y que además permite establecer un nexo directo entre la formación de palabras de MVNIDI y MVNITIE y la del ND PANDITI de la 1. 8. Hasta ahora carecemos de datos fiables acerca del resultado fonético y la representación gráfica de un dat. sg. temático de palabras provistas de un sufijo -yo- en inscrs. indígenas, con una excepción: en Lamas de Moledo, donde los dats. masc. terminan en $-<\mathrm{OI}>$ (MACAREAICOI, CAEILOBRIGOI), se atestigua una palabra PETRAVIOI o PETRANIOI. No faltan casos de $-<\mathrm{IO}>$ en epígrafes menores de la fachada occidental, si bien puede tratarse de formas latinizadas. En zonas geográficas donde el dat. masc. es $-<\mathrm{V}>0-<\mathrm{VI}>$, podría razonablemente esperarse testimonios de $-<\mathrm{IV}>$. Un dat. masc. en $-<\mathrm{IVI}>$ aparece sólo en la fórmula teonímica LARI COVTICIVI (Viseu, siglo I).

La forma MVNITIE podría ser precisamente un dat. masc. temático. Para ello se requiere suponer la evolución que sigue: dat. masc. ${ }^{*}-y \bar{o} i>$ abreviación condicionada del primer elemento de diptongo ${ }^{*}-y o \check{~} i>$ asimilación contextual $*$-yei > monoptongación *-ye. Hemos hablado ya de la secuencia AV[---] MVNITIE como ND + epíteto: esta forma encubriría entonces un epíteto *monèt-yo-, de función similar a la que cumplen las formas correspondientes de las fórmulas Iūno Monēta y, en Creta, Zev̀s Movvítıos, además del NL ilirio Movítıov. Esta constatación no restaría nada a las probabilidades de que MVNIDI y MVNITIE se puedan vincular etimológicamente y estén sincrónicamente relacionadas, la segunda como derivado de la primera, y apunta a que MVNIDI tiene todavía valor apelativo relacionado con cumbres y elevaciones, y no es un apelativo de valor genérico, como «divinidad» o «ninfa».

Adicionalmente, esto explicaría la forma HARASE del principio de la 1. 2. Esta forma debe tener alguna relación derivacional con AHARACVI y con HARACVI, y con el epíteto cortado (no abreviado) H[...] de BROENEIAE.

\footnotetext{
${ }^{16}$ Cf. Gorrochategui 1990; Rubio 2004.
} 
Se puede admitir en principio que el grafo $<\mathrm{S}>$ de HARASE representa el producto de la palatalización de la velar ante un sufijo -yo-, de la que hay ejemplos en el centro y sur de Portugal en los epítetos divinos CRONISENSI (Castelo Branco, de *koron-ik-yo-), SAISABRO (Portalegre, de *saikyo/a-bri-) o VASECO (Coimbra, de *waky-aiko-). Si estamos en lo cierto, HARASE es un derivado en -yo- a partir de otro adjetivo previo en -ako- (o -āko-), de manera que la secuencia *-ak-y-ōi ha sufrido los cambios *-akyŏi > *-akyĕi > *-akye > *-ǎse

Como hemos apuntado, es posible que la misma solución sea aplicable al caso de BROENEIAE, puesto que desconocemos la terminación del epíteto. Entonces habría que reconstruir un dat. masc. lusitano en ${ }^{*}-y-\bar{o} i$ con una evolución a $*-y \bar{e}$. Y la grafía $<\mathrm{AE}>$, única a lo largo del epígrafe, y en un dialecto y en una fecha en que no tenemos datos que avalen una tendencia a la monoptongación $-y a i->-y \bar{e}$-, sería tal vez producto de una interferencia con el uso gráfico latino, admitiendo que en latín los resultados de /e:/ y /ai/ empezaban a confundirse dando lugar a etacismo y grafías inversas. La explicación de este fenómeno está en línea con la del ac. sg. *-yŏm y el ac. pl. *-yŏns. Si la vocal /o/ tiende a sincoparse en la secuencia -yŏN(-) (y quizá -yŏs), no es raro que una vocal larga se haya abreviado en el mismo contexto, posiblemente ante cualquier consonante o segundo término de diptongo.

En todo caso, la alternativa es que $-<\mathrm{IE}>$ sea una representación peculiar de $*-y$-a $i$, dat. fem. de un derivado en $-y o-$, ya que esperaríamos en principio una representación ${ }^{* *}$ MVNITIA o ${ }^{* *}$ MVNITIAE. Obviamente, esto no se compadece con la aparición de un dat. fem. terminado en $-<A>$, como CARIA. Por tanto, CARIA es el único obstáculo que impide interpretar BROENEIAE, HARASE y MVNITIE como dats. fem. en *-y-āi. La alternativa a todo lo anterior, pues, consiste en asumir, como se apuntó arriba, que CARIA es una forma abreviada, y encubre un epíteto del ND MVNITIE. La secuencia completa sería MVNITIE CARIA(CAE) CANTIBIDONE, uel sim. Evidentemente, ésta es una respuesta poco satisfactoria a las inconsistencias gráficas del texto.

Por último, queda en la incógnita el resto gráfico mínimo que aparece, precedido de interpunción, tras CANTIBIDONE. Si excluimos que haya sitio para una ofrenda en ac. sg./pl., que obligaría a invertir el orden general ac. + dat. a favor de uno de dat. + ac. (cf. n. 8), restaría ignorar la aparente interpunción y leer CANTIBIDONEA, forma que, a su vez abreviada o no, concertaría con CARIA (cf. supra), o pensar en algún sello final del texto que no podemos deducir. 


\section{APINVS VENDICVS ERIACAINV[S]}

APINVS.- - Los NP APINVS y APINA son típicos de Lusitania y sobre todo de la Emeritensis y Scallabitana. No existe apenas documentación fuera de la Península, salvo un caso de APINVS y APINVLA en una inscr. funeraria de Auxerre, Francia (CIL XIII 2928) y un nomen latino Apinius al que pudiera concederse el mismo origen. Procede de IE $* H_{2} e p$ - 'agua' (cf. Albertos 1966, p. 1).

VENDICVS. - VENDIO (Navarra), VENDALO (Cuenca), VENDIECI (Orense), VENDIRICVS (Asturias, CIL II 5747), que procede de la asimilación a la flexión temática de un antiguo *wendi-rēg-s, NP todos ellos típicamente hispanos, posiblemente provenientes de *wendh- 'barba, pelo', de los cuales éste es el primer caso documentado que procede de Lusitania. No está claro, en cambio, que haya que relacionar VENDICVS con la Ribeira da $V e n d a$, de donde procede la inscripción, sobre todo porque, de tratarse de un único firmante, una expresión onomástica del tipo de $\mathrm{NP}^{1}+$ localicio $+\mathrm{NP}^{2}$ es desconocida y deja en suspenso la interpretación del incierto OVGVI[-]

ERIACAINVS. - Es un compuesto probablemente celta. Su primer elemento es *peri 'alrededor', que se documenta en otros NP de la Galia, como ERIDVBNOS, y en Italia Eripoxios (cf. $D L G$ ). Un dat. ACAINO se documenta en Ebersdorf (Baviera, CIL III 4595). Parece, además, recomendable la comparación con la forma Akainakubos (Botorrita I). Y el NP Acinus se documenta en Viseu (Portugal) y Moral de Sayago (Zamora) -cf. Vallejo 2005, p. 106-. Hainzmann y De Bernardo Stempel 2007 relacionan Akainakubos con gr. ók $\alpha \mathbf{v} \alpha$, de ${ }^{*} H_{2} e k-n$-, que designa en Tesalia una medida del terreno, y mencionan una divinidad ACINORIS cuyo primer elemento sería «das altkeltische Landmaß acina», presente en el NL bretón Aguénéac < Aciniācus o el suizo Acinnacum (a. 961), que son en realidad derivados de nomina possessoris. Pero esto no contribuye decisivamente a explicar la aparición de -acainus en un compuesto de carácter intensivo, que, a juzgar por la presencia del prefijo eri-, alude a una cualidad compartida por el portador del NP en grado elevado, como sucede en adjetivos de otras lenguas (IEW, p. 810), donde *peri se emplea con el valor intensivo de 'muy'. En consecuencia, postularemos la siguiente alternativa: el núcleo original de este NP es un compuesto celta * $(\varphi)$ eri-āku/o- 'muy rápido', antónimo de galo DIACVS 'lento, perezoso', de *dē-āku-/o- (DLG, p. 144). La adición del 
sufijo -(a)inus está avalada por dobletes como ABLECA, ABLECAENVS (Zamora, León), CAMALVS, CAMALAENVS (Braga).

\section{ICCINVI}

Maggi 1983 sugirió identificar el ND ICCONA LOIMINNA (Cabeço das Fráguas) con la gala Epona. Esto requeriría suponer resultados diferentes para labiovelares y segmentos $-k w$ - en el área occidental, como defiende Moralejo 1998. Aunque la aparición de nuevos documentos irá perfilando nuestras ideas sobre el particular, y tal vez haya que hablar de varios dialectos con soluciones distintas, se aprecia que el resultado de IE $/ \mathrm{k}^{\mathrm{w}} /$ es normalmente $/ \mathrm{p} /$. La secuencia $/ \mathrm{kw} /$ parece que da un resultado diferente, como en jón.-át. ï $\pi \pi \mathrm{o}$, tarentino $1 \kappa \kappa o \varsigma$. ICCONA habría experimentado un proceso de geminación *ekwo- > $e k k w o->*$ Ikko-, explicable por resilabación de una secuencia *-V.CwV- > -VC.CwV- seguida por la absorción de /w/ cuando precede una consonante geminada: *-VC.CwV- > -VC.CV-. En tal caso podría aceptarse la comparación con una inscr. votiva cuyo texto, de acuerdo con Marco 1999, es DEIS EQVEVNV(BO).

No puede descartarse que se trate en ambos casos de derivados de una base *icc- de etimología discutida, pero de amplia presencia en zonas de implantación celta (cf. $A c S$ II, 17-23). Por ejemplo, podría tratarse de la raíz *yeH $H_{1}$ - (cf. IEW, p. 504), bien documentada en las lenguas celtas. Ésta era la hipótesis original de Tovar 1967, y sólo se podría sustentar sobre la base de que se ha producido una evolución *yēk- > *yīk- > ìkk-. Por lo demás, la relación léxica de ICCONA con ICCINVI, añadida al hecho de que el epíteto LOIMINNA aparece en Arroyo de la Luz I bajo la forma LOEMINA, aunque sin ND expreso, remacha la imposibilidad de entender los ND en $-<\mathrm{A}>$ como nominativos y apunta a que se trata de dats. fem. en $-<\mathrm{A}>$.

\section{PANDITI}

Como en el caso de MVNIDI y su probable derivado MVNITIE, PANDITI parece una forma derivada con sufijo dental (cf. infra). Al ser nombre propio, podría ser de origen celta aunque aparezca en una inscr. de otra atribución dialectal. En tal caso, podría relacionarse con un grupo de NP compuestos ga- 
los con una secuencia -panto-, como VERPANTVS o AVIOPANTIS, que se atribuyen a la raíz * $k^{w}$ ent- 'sufrir' (cf. $D L G$, s. u.), aunque, si es celta nuestro $\mathrm{ND}$, parece más aconsejable acudir al étimo *kwnt- de formas como m.gal. pant 'pendiente, valle', el verbo denominativo antiguo bretón pantet, glosado imminet, o tal vez airl. céite 'asamblea' (si procede de * $k^{w} a n t-y \bar{a}$ y no de *kant-yā, cf. LEIA C-58), los ríos británicos Pont y Pant, el NL Pantiñobre (Coruña) y los NL alemanes Pfinz, de ${ }^{*} k^{w}$ ontina $\bar{a}$, y Künzing, de ${ }^{*} k^{w}$ entinā (cf. Geiger 1963). El obstáculo que presenta la aparente sonorización -nt- > -nd- en una forma celta, a pesar de que no se da en los resultados actuales o antiguos de formas celtas de Galicia, como Brigantium o Pantiñobre no es insuperable, dado que existe en celtibérico y que desconocemos el alcance de la fragmentación dialectal del celta peninsular. No obstante, la verosimilitud de que se trate de una divinidad local, unida a la conservación de -nt- en las inscrs. de la zona, que contienen p. ej. el localicio Cantibidonensis, hacen esta vinculación sospechosa. Y hemos observado que el análisis de la sufijación de PANDITI y MVNITIE hace probable que la base sea verbal y no nominal. Esto no afecta a la idea de que BANDI, divinidad no-celta y extendida desde el norte, proceda de * $g^{w}{ }_{0}$-tu- 'pasaje' (cf. $L R P$, cap. IX).

Existe, sin embargo, una opción más atractiva: que se trate de un derivado

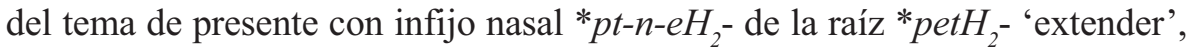
que se continúa por ejemplo en latín pando 'extender' y pateo 'abrirse, extenderse'. Existe también en el mundo religioso latino una Dea Panda ... uel Pantica, cuyo nombre nos ha llegado a través de Arnobio (Adu. Nat. 4.128), supuestamente una antigua divinidad de los caminos, que tenía la función de uiam pandere et aperire. Así, la etimología más probable de PANDITI contiene un nuevo caso de /p/ inicial, que imposibilita su atribución al grupo celta.

Ahora bien, la única manera de justificar que un nombre de agente en -etse forme sobre un tema de presente infijado es que este tipo en -et- se haya hecho productivo y que el paradigma verbal se haya nivelado, de modo que el infijo se haya extendido fuera del presente. Eso es lo que sucedió en lat. pando (perf. pandī, part. pas. passus o pansus). Este verbo suscita una serie de problemas relacionados con la sonorización de la dental y el vocalismo radical que nunca han sido convincentemente resueltos. Schrijver 1991, pp. 499-504, postula que un singular regularmente reconstruido $* p t-n-e H_{2}-t i$ sufrió tanto en itálico como en celta una serie de cambios, en virtud de los cuales: $a$ ) la dental sonorizó, dando * $p d-n-e H_{2}-t i ; b$ ) se produjo una metátesis que dio * $p n d e H_{2}-t i$; c) se extendió el grado cero radical en los presentes con infijo nasal, obtenién- 
dose así ${ }^{*} p n d H_{2}-t i$; ) se perdió la laringal, dando finalmente * $p n d t i$. A partir de aquí, un resultado itálico *pand- sería regular, aunque hay que explicar el vocalismo latino radical. En plural, si se parte de *pt-n $-H_{2}-e n t i$, no se habría producido neutralización de dentales en contacto con nasal, lo que explica la alternancia $d / t$ que se deduce de osco patensíns. La 3. ${ }^{a}$ pers. pl. es precisamente el punto de partida para $L I V$, p. 430 , que postula una secuencia evolutiva *p $p_{2}$-n $-H_{2}$-enti $>$ *patanant $>$ *patanont $>$ *patno- $>$ pando.

Todo esto suscita un problema ulterior, como es la relación del verbo pando con el adjetivo pandus 'curvo'. La Dea Panda se compara tradicionalmente con la Padella umbra, idéntica a la Patella latina, y con el dat. fem. osco en dativo Patanaí (Agnone). Subsisten problemas relacionados con el consonantismo. Si estas formas son antiguos participios pasivos en -(e)no-, comparables a avéstico paӨana-, entonces también osco patanaí puede explicarse como procedente de ${ }^{*} p_{\partial} t H_{2}$-eno-. La forma latina pandus y su divinización Panda vendrían del part. pas. ${ }^{*} p t H_{2}-n o-$ con pérdida muy primitiva de laringal y sonorización de -t $t$. La pérdida debería ser analógica de la que, hipotéticamente, se da en sílaba no inicial y entre oclusivas. Por tanto, habría que pensar que el antiguo part. * $\mathrm{ptH}_{2}-n o$ - salió del paradigma y fue reemplazado muy primitivamente por uno regular ${ }^{*} \mathrm{ptH}_{2}$-to-, y que al evolucionar éste a $* p_{2}$-to- con pérdida de laringal (y finalmente * passo-), se produjo analógicamente un resultado *pt-no-, luego *pd-no- y, con metátesis, *pndo-. Sumamente interesante es la divinidad ח $\alpha v \delta$ iv $\alpha$ del Bruttium, al extremo meridional de Italia, de la que no existe información, excepto porque su nombre aparece en monedas del siglo IV a. C. de las ciudades de Hipponium y Terina, con iconografía asociada que la representa con cetro. Heurgon 1972, p. 62, la considera como un ND protolatino, diminutivo de Panda, que habría sido identificada con Ceres en la zona griega de Italia, pero el conjunto de datos apunta a un ND de mayor antigüedad y dispersión dialectal.

Finalmente, Hamp 1977 postulaba una raíz * $p_{2} H_{2}$ nd- sobre la base de una correspondencia de itálico y celta. Ya Ó Briain 1923, p. 319, había comparado airl. és 'huella, sendero' con la forma lat. pansa (Plauto) 'de pie ancho o plano'. Hamp (p. 243) modifica esta comparación en la idea de que el part. lat. passum proviene regularmente de *pandto-. Del mismo modo, en airl. se habría dado la evolución *pandtā $>{ }^{*}$ pantsta $>*$ anss $\bar{a}>{ }^{*} \bar{\varepsilon} s s \bar{a}>$ és. Por consiguiente, el verbo pando no es el resultado de la nasalización de la raíz ${ }^{*} p e t H_{2}$, y de ahí el postulado de una raíz independiente. Esta idea sería de inmediata aplicación tanto a nuestro caso como a los otros ND. Más tarde, el mismo autor (Hamp 
1988) amplió esta hipótesis al NL británico Ad Ansam (Suffolk, It. Ant. IX 15 y Tab. Peut.), que reflejaría el mismo étimo. Recientemente, Irslinger 2002, p. 342, explica las formas celtas como producto de ${ }^{*}$ pent-te $H_{2}$, derivado de *pent- 'encontrar (un camino) ${ }^{17}$. En todo caso, es verosímil que PANDITI provenga del nombre de agente *pand-ê/ět- 'que (se) expande' o de un compuesto agentivo *pand(o)-i-t-.

\section{ATTEDIA}

NP fem. indígena en nom. sg., salvo error, documentado aquí por primera vez en Hispania. ATTEDIAE y ATTEDIVS aparecen en una misma inscr. de la Moesia Superior (Ravna, Serbia, cf. ILJug 1325), y hay otro ATTEDIAE en Narbona ${ }^{18}$. Se trata sin duda de un derivado en -ed-yo- de *atta, forma de origen expresivo que designa al padre biológico o al que en efecto cría al individuo. A juzgar por su distribución y paralelos es, probablemente, celta. Equivale etimológicamente al nomen Attidius.

\section{PVMPI}

Según todas las apariencias PVMPI representa el testimonio más occidental de un numeral ' 5 ' 19 . Si aceptamos que la parte inferior de la inscr. contiene una fórmula filiativa, PVMPI puede ser el genitivo de singular de un NP nunca antes documentado Pumpius, comparable al nomen de origen itálico Pompeius. Tanto en itálico como en celta existen formas con un grado /o/ *ponk $k^{w}$. Al norte de Italia se documenta un patronímico o NP celta Pompe-teguaios 'que tiene cinco lenguas' (Eska 1999). Documentan un apelativo fitonímico $\pi \varepsilon \mu \pi \varepsilon-\delta o v \lambda \alpha$, $\pi \mathrm{o} \mu \pi \varepsilon-\delta o v \lambda \alpha$ 'cinco-hojas' Dioscórides y Pseudo-Apuleyo (André 1985).

${ }^{17}$ Cf. IEW, pp. 808-809.

${ }^{18}$ Cf. Dellong 2002, núm. 89.

${ }^{19}$ Es el único caso hispano a excepción de los NP de *penkwt-yo- (cf. Villar 1996). El étnico EX GENT(E) PEMBELOR(VM) (CIL II 5729, Parres, Asturias) fue interpretado por Tovar como diminutivo de *pempe. Prósper 2008 propone un compuesto *penno-belo- 'de cabeza grande'. 


\section{RESUMEN DE LOS RASGOS FONÉTICOS Y MORFOLÓGICOS}

\section{Fonética}

1) Conservación de IE /p/ $\rightarrow$ PANDITI. Rasgo compartido, se da en toda la fachada occidental (PORCOM, PRAISOM, PRAESONDO, POEMANAE, PEMANEIECO, COROPOTI).

2) Vocalización - an- de la sonante $/ \mathrm{n} / \rightarrow$ PANDITI. Rasgo compartido con el resto de los testimonios no celtas de la fachada occidental, como en PEMANEIECO, QVANGEIO, BANDVE. Depende de la interpretación de la secuencia $<$ PAND $>-$.

3) Conservación de oclusivas sordas intervocálicas $\rightarrow$ IFATE, MVNITIE, PANDITI. Rasgo arcaizante, que contrasta con la tendencia a la sonorización de etapas posteriores y/o de zonas más septentrionales.

4) Pérdida de $/ \mathrm{w} /$ en posición intervocálica en contexto -owíle- $\rightarrow$ OILA, OILAM, posiblemente BROENEIAE. Rasgo compartido, conocido por Cabeço das Fráguas: OILAM.

5) Fricativización y ensordecimiento de labial sonora aspirada $/ \mathrm{bh} / \rightarrow$ IFATE. Rasgo compartido, se da en toda la fachada occidental en apelativos (IFADEM, SEFIO, FIDVENEARVM).

6) Fricativización y ensordecimiento de labial sonora aspirada $/ \mathrm{gh} /$, con signos gráficos de tendencia a la pérdida fonética $\rightarrow$ HARASE, HARACVI, AHARACVI. Como alternativa puede ser un resultado aspirado de /s/ en posición inicial y medial de la palabra, que no se contradice con su pérdida en posición final. Rasgo arcaizante si en otras zonas hay pérdida total de $/ \mathrm{gh} /$ que ya no llega a reflejarse en el sistema gráfico: VEAM en Arroyo de la Luz I puede venir de *wegh-yā 'animal de carga', cf ai. vahyá- 'vehículo', as. wigg 'caballo' ${ }^{20}$.

7) Pérdida de nasal ante fricativa $\rightarrow$ IFATE. Rasgo compartido al menos con Cabeço das Fráguas: IFADEM y con el ND COSSVE, COSO.

8) Palatalización del grupo $-k y->-\check{s}-($ representado como $<\mathrm{S}>$ ) $\rightarrow$ HARASE. Rasgo compartido con el centro y sur de Portugal (CRONISENSI, SAISABRO, VASECO).

\footnotetext{
${ }^{20}$ Esta forma podría estar en la inscr. rupestre perdida de Freixo de Numão (Guarda), que decía, según CIL II 430: IVNO(NI?) / VEAMVAEARVM / TARBOVM AN/CNVNARVM / SACRVM / CIRI / CVR(AVERVNT), con una serie de nombres de animales en ac. sg., donde leemos VEAM '¿animal de carga?', VAEARVM (?), TARBOVM ‘toro', ANCNVN ‘cordero' (lat. agnus, lusitano ANCOM), ARVM '¿campo?', forma latina o correspondencia lusitana de lat. aruum.
} 
9) Resultado asimilatorio del grupo de labial + labiovelar * $p-k^{w}>k^{w}-k^{w}$ $\rightarrow$ PVMPI.

10) Resultado labial de la labiovelar sorda $k^{w}-k^{w}>p-p \rightarrow$ PVMPI.

11) Cierre $/ \mathrm{o} />/ \mathrm{u} /$ en contacto con oclusivas o nasales labiales $\rightarrow$ MVNITIE, PVMPI. Rasgo compartido, se conoce por el área de expansión de MVNIDI, centro y sur de Portugal y Arroyo de la Luz I: PVPPID, ENVPETANIM, MVITIEAS.

12) Cierre /e:/ > /i:/ $\rightarrow$ PANDITI, MVNITIE (evolución meramente posible. La alternativa es partir de una sufijación -i-t(i)-). Rasgo compartido, por el centro y sur de Portugal.

13) Cierre /o:/ > /u:/ al menos en sílaba final $\rightarrow$ HARACVI, ICCINVI.

14) Síncopa de vocal temática tras -y-, ante nasal: -yŏm > -im. Rasgo compartido, se da en Arroyo de la Luz I: NVRIM, ENVPETANIM y Cabeço das Fráguas: IFADEM.

15) Neutralización de /e/, /i/ ante nasal: -ins $>$-ens $\rightarrow$ deducida de IFATE. Rasgo compartido, se da en Cabeço das Fráguas: IFADEM.

16) Reducción del grupo consonántico $-n s$ en posición final, con un resultado $-s \rightarrow$ deducido lógicamente de la explicación ofrecida para OILA, IFATE (v. punto 17).

17) Pérdida de /s/ en posición final $\rightarrow$ OILA, IFATE. Rasgo posiblemente único, frente a SECIAS, MVITIEAS (pero ante consonante ERBA) en Arroyo de la Luz I. Podría limitarse a cuando precede vocal larga o a posición anteconsonántica.

18) Reducción y monoptongación de diptongo largo después de $-y-:-y \bar{o} i$ $>-y \check{o} i>-y \breve{e} i>-y \bar{e} \rightarrow$ HARASE, MVNITIE, BROENEIAE (o alternativamente $-y \bar{a} i>-y a i>-y a e>-y \bar{e}$ ). Rasgo único, desconocido hasta la fecha, que se opone, al menos en sus dos últimos pasos, a la evidencia única de $-<\mathrm{IOI}>$ aportada por Lamas de Moledo y que depende de que CARIA/CAREA esté bien leído En el caso de que fuera abreviatura de *CARIA(CAE), estas formas podrían ser dats. fem. en $-\bar{a} i$ con monoptongación condicionada a su posición en sílaba final y al contacto con $-y$-.

\section{Morfología}

1) Dat. sg. masc. temático: $-<$ VI $>\rightarrow$ HARACVI, AHARACVI, ICCINVI. Rasgo compartido con el sur y centro de Portugal.

Posible dat. sg. masc. temático (o tal vez femenino) en $-<\mathrm{E}>$ de los temas en -yo-: $\rightarrow$ HARASE, MVNITIE, BROENEIAE. Rasgo único. 
2) Dat. sg. fem. en $-<A>\rightarrow$ CARIA. Rasgo compartido. Los dats. en $-<A>$ se concentran en una zona que va desde la hoz del Mondego al sur de la confluencia del Tajo y el Alagón, con esporádicas extensiones hacia norte y sur.

3) Dat. sg. atemático en $-<\mathrm{I}>\mathrm{o}-<\mathrm{E}>\rightarrow$ BANDI, PANDITI vs REVE. Rasgo compartido con toda la fachada occidental en el caso de REVE, con Lusitania en el de BANDI.

\section{BIBLIOGRAFÍA}

AcS $=$ Holder, A. 1896-1922: Alt-celtischer Sprachschatz I-III, Leipzig.

Albertos, M. ${ }^{a}$ L. 1966: La onomástica personal primitiva de Hispania: Tarraconense y Bética, Salamanca, CSIC.

André, J. 1985: «Noms de plantes gaulois ou prétendus gaulois dans les textes grecs et latins», Études Celtiques 22, pp. 179-198.

Carneiro, A., Encarnação, J., Oliveira, J. y Teixeira, C. 2008: «Uma inscrição votiva em língua lusitana», Palaeohispanica 8, pp. 1-11.

Dellong, E. 2002: Narbonne et le Narbonnais 11/1. Carte archéologique de la Gaule, París, MSH.

$D L G=$ Delamarre, X. 2002²: Dictionnaire de la langue gauloise, París, Errance.

Eska, J. F. 1999: «The linguistic mileu of *Oderzo 7», HS 112, pp. 122-136.

Geiger, Th. 1963: «Die ältesten Gewässernamen-schichten im Gebiet des Hoch- und Oberrheins (Fortsetzung)», BNf 16, pp. 233-263.

Gorrochategui, J. 1990: «Consideraciones sobre la fórmula onomástica y la expresión de origen en algunos textos celtibéricos menores», en Villar, F. (ed.), Studia indogermanica et palaeohispanica in honorem A. Tovar et L. Michelena, Salamanca, Universidad del País Vasco-Universidad de Salamanca, pp. 291-312.

Hainzmann, M. y De Bernardo Stempel, P. 2007: «Zwei neue keltische Gottheiten: Ollodevos und Acinoris», en Hainzmann, M. (ed.), Akten des 5. F.E.R.C.AN.Workshop, Viena, Verlag der Österreichischen Akademie der Wissenschaften, pp. 139-146.

Hamp, E. P. 1977: «Some Italic and Celtic correspondences», KZ 91, pp. 240-245.

- 1988: «Ad Ansam», BBCS 35, pp. 54-55.

Heurgon, J. 1972: «I culti non greci della Magna Grecia», Atti del XI Convegno di Studi sulla Magna Grecia, Nápoles, pp. 55-75.

IEW = Pokorny, J. 1959: Indogermanisches Etymologisches Wörterbuch, Berna, Francke.

ILJug = Šašel, A. y Šašel, J. 1963-1986: Inscriptiones Latinae quae in Jugoslavia inter annos MCMII et MCMXL repertae et editae sunt, Lubliana, SAZU.

Irslinger, B. S. 2002: Abstrakta mit Dentalsuffixen im Altirischen, Heidelberg, Winter. 
Jordán Cólera, C. 2005: «¿Sistema dual de escritura en celtibérico?», Palaeohispanica 5, pp. 1013-1030.

LEIA = Vendryes, J., Bachellery, E. y Lambert, P.-Y. (eds.) 1959 ss.: Lexique étymologique de l'irlandais ancien, París, CNRS.

$L I V=$ Rix, H. et alii 1998: Lexikon der indogermanischen Verben, Wiesbaden, Dr. Ludwig Reichert Verlag.

$L R P=$ Prósper, B. M. ${ }^{\text {2 } 2002: ~ L e n g u a s ~ y ~ r e l i g i o n e s ~ p r e r r o m a n a s ~ d e l ~ O c c i d e n t e ~ d e ~ l a ~}$ Península Ibérica, Salamanca, Ediciones Universidad de Salamanca.

Maggi, D. 1983: «Sui teonimi Trebopala e Iccona nell'iscrizione lusitana del Cabeço das Fraguas», en Campanile, E. (ed.), Problemi di Lingua e Cultura nel Campo Indoeuropeo, Pisa, Giardini, pp. 53-60.

Marco, F. 1999: «Deis Equeunu(bo)», en Villar, F. y Beltrán, F. (eds.), pp. 481-490. Mayer, A. 1957-1959: Die Sprache der alten Illyrier I-II, Viena, ÖAkW.

MLH IV = Untermann, J. 1997: Monumenta Linguarum Hispanicarum IV. Die tartessischen, keltiberischen und lusitanischen Inschriften (unter Mitwirkung von D. Wodtko), Wiesbaden, Dr. Ludwig Reichert Verlag.

Moralejo, J. J. 1998: Labiovelares en material galaico y lusitano, Ferrol, el autor.

Nieto, E. 1993: «Remarques sur le prétendu datif singulier en - $a$ dans le latin archaïque», IF 98, pp. 155-176.

Ó Briain, M. 1923: «Hibernica», ZCPh 14, pp. 309-334.

Pedrero, R. M. ${ }^{a}$ 1999: "Aproximación lingüística al teónimo lusitano-gallego Bandue/Bandi», en Villar, F. y Beltrán, F. (eds.), pp. 535-543.

— 2001: «Los epítetos del teónimo lusitano-gallego Bandue/Bandi», en Villar, F. y Fernández Álvarez, M. ${ }^{a}$ P. (eds.), pp. 541-560.

Prósper, B. M. ${ }^{\text {a } 2004: ~ « V a r i a ~ P a l a e o h i s p a n i c a ~ O c c i d e n t a l i a », ~ P a l a e o h i s p a n i c a ~ 4, ~}$ pp. 169-194.

— 2008: «Los nombres “itálicos” de los astures meridionales», Conimbriga 47, pp. 145-69.

$R A P=$ Garcia, J. M. 1991: Religiões antigas de Portugal, Lisboa, Imprensa Nacional-Casa da Moeda.

Rieken, E. 1999: Untersuchungen zur nominalen Stammbildung des Hethitischen, Wiesbaden, Reichert.

Rubio, F. 2004: «La tésera celtibérica de Sasamón (K.14.1)», Emerita 72, pp. 121153.

Salas, J., Esteban, J. y Rueda, G. 1989: «Bandia Apolosegus, una divinidad con culto local en la zona de Brozas, Cáceres», $H A$ 13, pp. 7-20.

Schrijver, P. 1991: The reflexes of the Proto-Indo-European laryngeals in Latin, Ámsterdam-Atlanta, Rodopi.

Tovar Llorente, A. 1954: «Numerales indoeuropeos en Hispania», Zephyrus 5, pp. 17-22. 
Tovar Llorente, A. 1967: «L'inscription du Cabeço das Fráguas et la langue des lusitaniens», ÉC 11, pp. 237-268.

Untermann, J. 2000: Wörterbuch des Oskisch-Umbrischen, Heidelberg, Carl Winter Verlag.

Vallejo Ruiz, J. M. ${ }^{a}$ 2005: Antroponimia indígena de la Lusitania romana, Vitoria, Universidad del País Vasco.

Villar, F. 1981: Dativo y locativo en el singular de la flexión nominal indoeuropea, Salamanca, Ediciones Universidad de Salamanca.

- 1985: «El dativo temático indoeuropeo», en Melena, J. L. (ed.), Symbolae Ludovico Mitxelena Septuagenario Oblatae, Vitoria, pp. 31-48.

- 1986: «El dativo latino epigráfico en -a», Emerita 54, pp. 45-62.

- 1994: «Los antropónimos en Pent-, Pint- y las lenguas indoeuropeas prerromanas de la Península Ibérica», en Bielmeier, R. y Stempel, R. (eds.), Indogermanica et Caucasica. Festschrift für K. H. Schmidt, Berlín-Nueva York, pp. 234-264.

- 1996: «El teónimo lusitano Reve y sus epítetos», en Meid, W. y Anreiter, P. (eds.), Die Grösseren Altkeltischen Sprachdenkmäler, Innsbruck, pp. 160-211.

Villar, F. y Beltrán, F. (eds.) 1999: Pueblos, Lenguas y Escrituras en la Hispania Prerromana. Actas del VII Coloquio de lenguas y culturas paleohispánicas, Salamanca, Ediciones Universidad de Salamanca.

Villar, F. y Fernández Álvarez, M. ${ }^{a}$ P. (eds.) 2001: Religión, Lengua y Cultura Prerromanas de Hispania, Actas del VIII Coloquio de lenguas y Culturas Paleohispánicas, Salamanca, Ediciones Universidad de Salamanca.

Villar, F. y Pedrero, R. 2001: «La nueva inscripción lusitana: Arroyo de la Luz III», en Villar, F. y Fernández Álvarez, M. ${ }^{a}$ P. (eds.), pp. 663-698.

Villar, F. y Untermann, J. 1999: «Las téseras de Gadir y Tarvodurum», en Villar, F. y Beltrán, F. (eds.), pp. 719-732.

Fecha de recepción de la primera versión del artículo: 31/10/2008

Fecha de aceptación del artículo: 06/11/2008

Fecha de recepción de la versión definitiva del artículo: 05/03/2009 